\title{
Efectividad y tolerabilidad de tres tipos de productos de preparación para colonoscopia
}

\section{Effectiveness and tolerability of three types of colonoscopy preparation products}

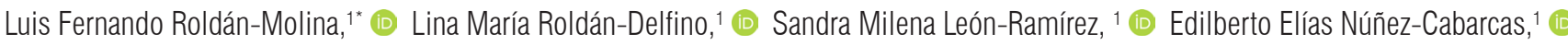

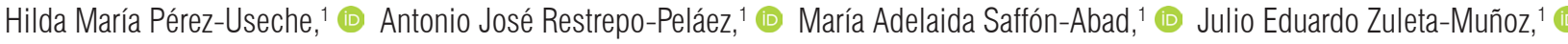 \\ Juan Nicolás Zuluaga-Aguilar. ${ }^{1}$ -
}

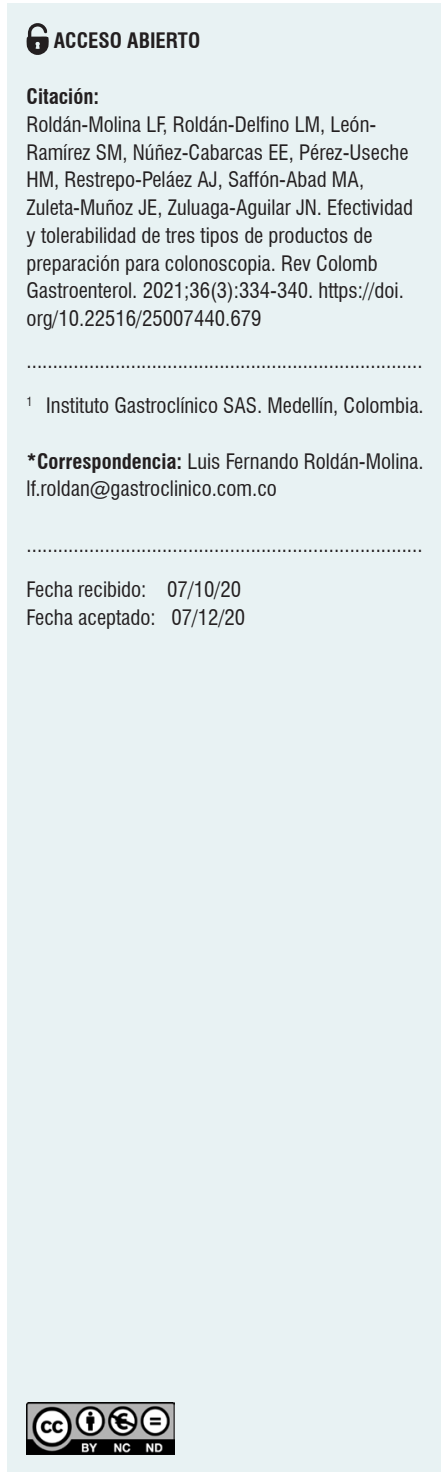

\section{Resumen}

Objetivo: establecer las diferencias entre tres tipos de productos de preparación para colonoscopia, en cuanto a efectividad y tolerabilidad. Materiales y métodos: estudio de corte transversal, analítico, prospectivo, ciego para el evaluador de la escala de Boston. Se incluyeron pacientes adultos, mayores de 18 años con requerimiento de colonoscopia y diligenciamiento de la encuesta sobre el tipo de preparación realizada para la limpieza del colon. Resultados: se evaluaron 3 grupos de productos (polietilenglicol, picosulfatos, sales de sulfato) en 907 pacientes, se aplicó la escala de Boston total y por segmentos, sin encontrar diferencias estadísticamente significativas entre ellos (Odds ratio [OR]: 1,10; intervalo de confianza [IC] $95 \%$ : 0,6-1,8; $p$ $=0,728)$. El $60 \%$ de la población fueron mujeres y la edad promedio 52 años. Se observó el cumplimiento de la dieta en el $99 \%$ de los participantes. La preparación dividida tuvo mejores resultados en la escala de Boston (OR: 5,06; IC $95 \%$ : 3,2-8,01; $p=0,001$ ). Los picosulfatos tuvieron mayor aceptabilidad (OR: 15,8; IC $95 \%: 8,83-28,3 ; p=0,001)$ y menores efectos secundarios como distensión abdominal (OR: 0,59; IC $95 \%$ : $0,3-0,9 ; p=0,033$ ) y vómito (OR: 0,25; IC $95 \%: 0,07-0,82 ; p=0,015$ ). Se observó mejor resultado cuando se realizó el examen antes de 6 horas de finalizada la preparación (OR: 6,38; IC 95 \%: 3,84-10,6; $p=0,001$ ). Conclusiones: los productos evaluados no presentaron diferencias entre sí con respecto a su efectividad. Los picosulfatos tuvieron menores efectos secundarios y mejor aceptabilidad. Se obtuvo una mejor preparación del colon con preparación dividida y si el examen es hasta 6 horas de finalizada la preparación.

\section{Palabras clave}

Colonoscopia, laxantes, efectos colaterales.

\begin{abstract}
Objective: To establish the differences between three types of colonoscopy preparation products in terms of effectiveness and tolerability. Materials and methods: An analytical, prospective, blind, cross-sectional study of the Boston Bowel Preparation Scale was carried out. Adult patients over 18 years of age with a requirement for colonoscopy and completion of the survey on the type of preparation carried out for colon cleansing were included. Results: Three groups of products (polyethylene glycol, picosulfates, and sulfate salts) were evaluated in 907 patients. Total and segment Boston Bowel Preparation Scale was applied, without finding statistically significant differences between them (OR 1.10;95\% Cl: $0.6-1.8 ; p=0.728) .60 \%$ of the population were women and the average age was 52 years. Compliance with the diet was observed in $99 \%$ of the participants. Split-dose bowel preparation performed best on the Boston scale $(\mathrm{OR} 5.06$; $95 \% \mathrm{Cl} ; 3.2-8.01$; $p=$ 0.001). Picosulfates had greater acceptability (OR 15.8; 95\% Cl: 8.83-28.3; $p=0.001)$ and fewer side effects such as abdominal distension (OR $0.59 ; 95 \% \mathrm{Cl}: 0.3-0.9 ; p=0.033$ ) and vomiting (OR 0.25; 95\% Cl: 0.07-0.82; $p=0.015)$. The best result was observed when the test was performed within 6 hours of completion of preparation (OR 6.38; 95\% Cl: 3.84-10.6; $p=0.001)$. Conclusions: The products evaluated did not show differences between them regarding their effectiveness. Picosulfates had fewer side effects and better acceptability. Splitdose and testing up to 6 hours after preparation resulted in better bowel preparation.
\end{abstract}

\section{Keywords}

Colonoscopy; Laxatives; Side effects. 


\section{INTRODUCCIÓN}

La visualización de la mucosa es fundamental en la detección de lesiones durante una colonoscopia y para tal fin se requiere una limpieza del colon buena o excelente ${ }^{(1,2)}$. La eficacia de la limpieza se evaluó a partir de la escala de Boston (Tabla 1), la cual ha sido validada internacionalmente y es de amplio uso en gastroenterología ${ }^{(2-4)}$. La escala representa la suma del puntaje de 0 a 3 , en los 3 segmentos del $\operatorname{colon}^{(4)}$, y se considera satisfactorio un puntaje $\geq 2$ en cada segmento ${ }^{(5)}$. Puntajes inferiores no reflejan una buena visualización y con ello disminuye la detección de adenomas y la intubación cecal ${ }^{(6,7)}$.

Para conseguir una buena preparación se han recomendado dietas bajas en fibra y residuos, sumado a una dieta líquida ${ }^{(8-10)}$, pero aun así se consideran medidas insuficientes. En consecuencia, se utilizan diversos tipos de catárticos y laxantes ${ }^{(8)}$, y los de mayor recomendación son aquellos laxantes de tipo osmótico a base de polietilenglicol (PEG), sales de sulfatos y picosulfatos ${ }^{(9,11,12)}$, todos reconocidos por sus resultados para la preparación del colon, pero con diferencias entre sí en cuanto a la forma de uso, tolerabilidad y recomendaciones de seguridad ${ }^{(13,14)}$.

El objetivo del estudio busca establecer las diferencias entre tres tipos de productos de preparación para colonoscopia, en cuanto a efectividad y tolerabilidad. El impacto de los productos a nivel de cambios hidroelectrolíticos ${ }^{(9,15)}$ exige un abordaje adicional, el cual no hace parte del alcance contemplado en el presente estudio.

\section{MATERIALES Y MÉTODOS}

Estudio de corte transversal, ciego para el evaluador de la escala de Boston. Se incluyeron todos los pacientes adul- tos, ambulatorios ${ }^{(16,17)}$ atendidos en una institución de gastroenterología de Medellín, Colombia, que se realizaron colonoscopia completa entre febrero y julio de 2020. Se construyó una base de datos en formato Excel, en el que se recogieron los siguientes datos de cada paciente para el análisis: edad, sexo, cumplió la dieta el día anterior, producto utilizado para la preparación, realizó la preparación dividida en dos partes, hora de terminación del producto, hora del examen y cómo le pareció el sabor del producto. Posteriormente, se adicionó a la base de datos los resultados de la escala de Boston total y por segmentos, de acuerdo con lo descrito en el informe de colonoscopia.

En el momento de la asignación de la cita para la colonoscopia se entregaron tres formatos con las diferentes instrucciones de preparación protocolizadas institucionalmente, para que el paciente seleccionara el producto de su preferencia (PEG, picosulfatos, sales de sulfatos). A todos se les recomendó una dieta sin semillas, cáscaras, leguminosas y legumbres, los dos días previos al examen y hasta la hora de iniciar la preparación. El grupo de pacientes preparados con PEG tomó 4 sobres diluidos en 1 litro de agua cada uno; el de picosulfatos tomó 2 sobres diluidos en $250 \mathrm{~mL}$ de agua cada uno y adicionalmente 1,5 litros de líquidos claros; y el grupo de sales de sulfato tomó dos frascos diluidos en $500 \mathrm{~mL}$ de agua cada uno y, adicionalmente, 1,5 litros de líquidos claros. A todos los pacientes se les recomendó realizar la preparación dividida en dos partes: la primera, a las 6:00 p. m. del día anterior al examen y la segunda, 5 horas antes de la hora programada para el examen, debiendo terminarla al menos 3 horas antes del procedimiento. Se indagó con respecto a la presencia de efectos secundarios relacionados con la ingesta de la preparación tales como distensión abdominal, cefalea y vómito. Los pacientes categorizaron su percepción del sabor del producto ingerido en agradable, indiferente o desagradable.

Tabla 1. Escala de preparación intestinal de Boston (por segmento de colon) ${ }^{(3)}$

Descripción

0 Segmento de colon no preparado con mucosa no visible debido a heces sólidas que no se pueden eliminar

1 Visibilidad de una porción de mucosa del segmento de colon, pero otras áreas del segmento no visibles debido a tinción, heces residuales o líquido opaco

2 Cantidad mínima de tinción residual, pequeños fragmentos de heces o líquido opaco, pero buena visibilidad de la mucosa del segmento de colon

3 Buena visibilidad de toda la mucosa del segmento de colon, sin tinción residual, pequeños fragmentos de heces o líquido opaco
Otras características de la escala

Rango de puntuación total (obtenida sumando puntuaciones para cada segmento):

Mínimo 0 (muy pobre) a máximo 9 (excelente) Puntuación realizada después de lavar o aspirar Segmentos clasificados por separado: colon derecho (incluido ciego y colon ascendente), transversal (incluye ángulos hepáticos y esplénicos) y colon izquierdo (colon descendente y sigmoide y recto)

El umbral óptimo es una puntuación total de $\geq 6$ y $\geq 2$ por segmento

Tomada de: Kastenberg D et al. World J Gastroenterol. 2018;24(26):2833-2843. 
La base de datos fue diligenciada por una asistente entrenada para tal fin en el momento de la admisión del paciente para la realización del procedimiento; registrando la información de tipo subjetivo no detallada en la historia clínica: cumplimiento de la dieta, producto utilizado, preparación dividida en dos partes o tomada en forma continua, hora de terminación del producto y percepción del sabor del producto.

La efectividad de la limpieza del colon se clasificó mediante la escala de Boston, la cual evalúa la presencia de deposiciones y visibilidad de la mucosa del colon en sus tres segmentos: derecho, transverso e izquierdo ( 0 a 3 puntos), y total ( 0 a 9 puntos). Se consideró una preparación inadecuada cuando en algún segmento la puntuación fue de 0 o 1 , o la puntuación total fue menor de 6. Dicha evaluación fue realizada por el grupo de gastroenterólogos que participó en la realización de colonoscopias, el cual está compuesto por 7 especialistas, y dicha evaluación quedó registrada en el reporte del examen. Se cuantificó el tiempo transcurrido entre el final de la ingesta del producto y la realización del examen con el fin de determinar el efecto en la preparación del colon y su consecuente visibilidad de la mucosa.

Los objetivos del estudio fueron determinar las diferencias en la preparación del colon con los diferentes productos de acuerdo con la escala de Boston, la diferencia entre la toma de la preparación en forma continua o dividida con referencia a la limpieza del colon y, a su vez, establecer resultados en cuanto a efectos secundarios, aceptabilidad del producto y tiempo transcurrido para la realización del examen posterior a la terminación de la ingesta con referencia al estado de la limpieza del colon.

Los productos evaluados hacen parte del grupo de laxantes osmóticos utilizados convencionalmente para este tipo de procedimientos; por tanto, no constituye una intervención experimental. No se realizó aleatorización de pacientes o demanda inducida para el procedimiento o los productos de preparación. Todos los pacientes cuentan con consentimiento informado para la realización del procedimiento. La conducción del estudio se considera de riesgo mínimo y contempla los principios fundamentales de la ética en investigación acordes a la Declaración de Helsinki versión $2013^{(18)}$ y a la Resolución 008430 de 1993 del Ministerio de Salud de Colombia ${ }^{(19)}$. El protocolo fue avalado previamente por el Comité de ética de la institución y se resguardó la confidencialidad de la información recogida.

\section{ANÁLISIS ESTADÍSTICO}

Los datos se analizaron con los programas Excel, versión 2019, y Jamovi, versión 1.2.25. Se realizó el análisis univariado en el que se determinaron frecuencias absolutas y relativas para las variables cualitativas. Para las variables

cuantitativas, se utilizaron media y desviación estándar (DE) o mediana y rango intercuartílico (RIC), previa verificación del supuesto de normalidad.

Se dicotomizaron las variables cuantitativas para realizar la comparación de proporciones. Se utilizó la prueba de asociación de chi cuadrado para muestras independientes y se estimó el Odds ratio (OR) con su respetivo intervalo de confianza (IC) del $95 \%$. Se consideró un valor de $p$ estadísticamente significativo $<0,05$.

\section{RESULTADOS}

Se identificaron 907 pacientes elegibles de un total de 1000 pacientes que fueron sometidos a colonoscopia entre febrero y julio de 2020. Se excluyeron de la base de datos los pacientes en los que no se realizó examen completo de todos los segmentos del colon, debido a dificultades anatómicas (angulaciones, adherencias o tumores obstructivos), alcance del estudio (antecedentes quirúrgicos con resección proximal del colon) o indicación de examen parcial (colonoscopia izquierda). Igualmente, se excluyeron a aquellos que realizaron la preparación con otro tipo de productos no protocolizados por la institución y aquellos en los que no se registró por el especialista la evaluación de la escala de Boston en el informe del examen.

El 60 \% de la población era de sexo femenino. La edad promedio fue de 52 años (DE: 14) (Tabla 2). La dieta baja en residuos fue seguida por el $99 \%$ de los pacientes. La preparación del colon se evaluó mediante la escala de Boston, la cual tiene un rango de 0 a 9 puntos (valoración total del colon) y de 0 a 3 puntos (valoración por segmentos).

Tabla 2. Características demográficas

\section{Variable}

Edad promedio

Mediana

Sexo femenino

Sexo masculino

Razón por sexo (mujer:hombre)

Fuente: propia.

Los productos protocolizados para la preparación del colon y que fueron sometidos a comparación entre sí son el PEG, picosulfatos y sales de sulfato. No se encontraron diferencias significativas entre los productos en cuanto a los resultados en la escala de Boston total o por segmentos (OR: 1,10; IC 95 \%: 0,6-1,8; $p=0,72$ ) (Tabla 3). 
Tabla 3. Efectividad y tolerabilidad según los productos de preparación de colon

\begin{tabular}{|c|c|c|c|c|c|c|}
\hline Variables & 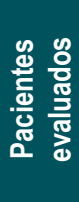 & 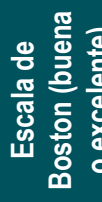 & 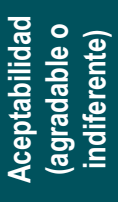 & 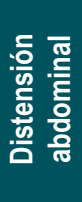 & 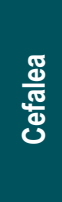 & 율 \\
\hline & $\mathbf{n}$ & $\%$ & $\%$ & $\%$ & $\%$ & $\%$ \\
\hline Picosulfatos & 196 & 90,3 & 93,4 & 11,2 & 16,3 & 1,5 \\
\hline PEG & 524 & 89,1 & 47,9 & 17,4 & 13,2 & 6,1 \\
\hline $\begin{array}{l}\text { Sales de } \\
\text { sulfato }\end{array}$ & 187 & 90,4 & 44,9 & 18,2 & 10,7 & 4,8 \\
\hline
\end{tabular}

Fuente: propia.

La indicación de ingerir la preparación dividida en dos tomas fue seguida por el $66 \%$ de pacientes y se obtienen mejores resultados en limpieza del colon que aquellos que la tomaron de forma continua (OR: 5,06; IC $95 \%$ : 3,2$8,01 ; p=0,001)$ (Tabla 4).

Tabla 4. Resultados de la escala de Boston según la forma de ingesta del producto de preparación y tiempo entre la preparación y el examen

\begin{tabular}{|lcc|}
\hline \multicolumn{1}{c}{ Variables } & $\begin{array}{c}\text { Pacientes } \\
\text { evaluados }\end{array}$ & $\begin{array}{c}\text { Escala de } \\
\text { Boston buena } \\
\text { o excelente }\end{array}$ \\
\cline { 2 - 3 } & $\mathbf{n}$ & $\%$ \\
\hline Preparación dividida & 602 & 95 \\
\hline Preparación continua & 305 & 79 \\
\hline Rango fin de preparación/examen $<6 \mathrm{~h}$ & 544 & 96,1 \\
\hline Rango fin de preparación/examen $>6 \mathrm{~h}$ & 358 & 79,6 \\
\hline
\end{tabular}

Fuente: propia.

La escala para evaluar el sabor del producto contó con tres opciones de respuesta: agradable, desagradable o indiferente. Las respuestas positivas (agradable) y neutras (indiferente) se trataron como aceptabilidad. El grupo de picosulfatos obtuvo mayor aceptabilidad (agradable: $77 \%$, indiferente: $16 \%$ ) respecto a los demás grupos (OR: 15,8; IC $95 \%$ : $8,8-28,3 ; p=0,001)$. Igualmente, presentó menores efectos secundarios tales como distensión abdominal (OR: 0,59; IC $95 \%: 0,3-0,9 ; p=0,03$ ) y vómito (OR: 0,25; IC $95 \%: 0,07-$ $0,82 ; p=0,015)$. En cuanto a la cefalea, no se encontraron diferencias significativas entre los productos evaluados.

El tiempo trascurrido entre la terminación del producto de preparación y el inicio del examen fue analizado segmen- tando la variable en 3 rangos de tiempo ( 0 a 6 horas, 6 a 12 horas, mayor de 12 horas), con el objetivo de identificar diferencias en el resultado de la preparación del colon. Se obtuvo que el rango con mejor resultado según la escala de Boston fue de 0 a 6 horas (OR: 6,38; IC $95 \%: 3,84-10,6 ; p=0,001$ ).

De los pacientes con estreñimiento $(n=69)$, el $90 \%$ tuvo una buena preparación.

\section{DISCUSIÓN}

Se realizó un estudio prospectivo con el objetivo de comparar la efectividad y tolerabilidad de tres tipos de productos para la limpieza del colon en colonoscopia ${ }^{(20)}$. La incidencia de inadecuada preparación fue del $10 \%$ en los pacientes evaluados, a diferencia de lo estimado en otros estudios, alrededor del $20 \%-40 \%{ }^{(21,22)}$. Esta diferencia puede deberse a la educación individualizada que se ofrece a los pacientes durante la asignación de la cita y dos días previos al procedimiento ${ }^{(23,24)}$. Los pacientes con mala preparación fueron principalmente mujeres (59\%) y personas menores de 60 años (68\%), lo cual controvierte algunos factores predictores de mala preparación reportados recientemente ${ }^{(21,25,26)}$.

La efectividad de la preparación se encuentra influenciada por la tolerabilidad del producto. Idealmente, el producto de limpieza para colonoscopia debería ser seguro, efectivo y bien tolerado ${ }^{(27,28)}$. La efectividad de los tres tipos de productos no presentó diferencias significativas entre sí. Resultados similares se han encontrado al comparar picosulfatos y $\mathrm{PEG}^{(29-31)}$. Cabe resaltar que no se indagó sobre el efecto de los productos en inflamación intestinal ${ }^{(32)}$.

Se obtuvo una mejor tolerabilidad en los pacientes que se prepararon con picosulfatos, presentando menor incidencia de distensión abdominal y vómito ${ }^{(11,31,33,34)}$. El sabor puede influir en la ingesta de la totalidad de la preparación e indirectamente en los resultados de la escala de Boston; al respecto, la mayoría de los pacientes manifestó percibir el sabor de los picosulfatos como agradable o indiferente. Tanto los PEG como las sales de sulfatos tuvieron baja aceptabilidad del sabor ${ }^{(9,11)}$.

Las restricciones de la dieta hacen parte de la preparación del colon; y es frecuente que muchos pacientes no sigan estas recomendaciones adecuadamente ${ }^{(35)}$. En contraste, los pacientes del estudio aceptaron seguir la dieta recomendada baja en residuos ${ }^{(36)}$, en el $99 \%$ de los casos. La hidratación adecuada también se incluye en todos los protocolos de preparación con el fin de evitar efectos fisiológicos adversos relacionados con la deshidratación como hipotensión o alteraciones hidroelectrolíticas ${ }^{(37,38)}$.

El uso de la preparación dividida fue significativamente mejor comparado con la ingesta continua ${ }^{(39,40)}$, independientemente del tipo de producto utilizado. El resultado es 
una mejor limpieza y visibilidad de la mucosa en todos los segmentos del colon ${ }^{(41,42)}$.

Diversos estudios sugieren que existe una ventana de tiempo después de finalizar la preparación y antes de que la limpieza del colon empiece a deteriorarse. El resultado obtenido fue un tiempo límite de 6 horas $^{(10,43)}$. Adicionalmente, al finalizar la ingesta del laxante mínimo 3 horas antes del procedimiento, se disminuye el riesgo de aspiración pulmonar relacionado con altos volúmenes gástricos residuales $^{(44)}$. Cabe resaltar que la dosis dividida también influye en los buenos resultados de la preparación debido a que el intervalo de tiempo entre la última ingesta del producto y la colonoscopia se hace más corto ${ }^{(40,45)}$.

Es importante indagar sobre los factores de riesgo independientes que afectan la preparación para colonoscopia, tales como estreñimiento, diabetes, demencia, cirugía colorrectal, sobrepeso, edad, entre otros, tanto en el paciente ambulatorio como en el hospitalizado ${ }^{(21,25,46)}$. En términos de seguridad, cualquiera de los productos puede tener efectos adversos. Se debe tener especial cuidado en aquellos pacientes con afectaciones cardíacas, hepáticas y renales $^{(47,48)}$. Es así como el conocimiento de las ventajas y desventajas de todos los productos permitirá una mejor selección para cada paciente ${ }^{(49)}$.

Para los pacientes con estreñimiento, el porcentaje de buena preparación fue similar al encontrado en la población del estudio. No se realizó un análisis estratificado según las comorbilidades o el uso de medicamentos relacionado con la efectividad de la preparación. Se recomienda que las próximas investigaciones aborden estas variables para el análisis.

\section{CONCLUSIONES}

Los productos evaluados no presentaron diferencias entre sí con respecto a su efectividad. Los picosulfatos tuvieron menores efectos secundarios y mejor aceptabilidad. Se obtuvo una mejor preparación del colon con preparación dividida y si el examen es hasta 6 horas de finalizada la preparación.

\section{Agradecimientos}

Al Doctor José Bareño por sus aportes durante la fase del análisis estadístico.

\section{REFERENCIAS}

1. Murcio-Pérez E, Téllez-Ávila F. Opciones de preparación para colonoscopia. Endoscopia. 2012;24(1):23-31.

2. Alvarez-Gonzalez MA. Same-Day Bowel Preparation: Excelling Bowel Cleansing Efficacy in Selected Outpatients. Dis Colon Rectum. 2019;62(12):1409-1410. https://doi.org/10.1097/DCR.0000000000001500

3. Kastenberg D, Bertiger G, Brogadir S. Bowel preparation quality scales for colonoscopy. World J Gastroenterol. 2018;24(26):2833-2843. https://doi.org/10.3748/wjg.v24.i26.2833

4. Seo M, Gweon TG, Huh CW, Ji JS, Choi H. Comparison of Bowel Cleansing Efficacy, Safety, Bowel Movement Kinetics, and Patient Tolerability of Same-Day and SplitDose Bowel Preparation Using 4L of Polyethylene Glycol: A Prospective Randomized Study. Dis Colon Rectum. 2019;62(12):1518-1527. https://doi.org/10.1097/DCR.0000000000001499

5. Gimeno-García AZ, de la Barreda Heuser R, Reygosa C, Hernandez A, Mascareño I, Nicolás-Pérez D, Jiménez A, Lara AJ, Alarcon-Fernández O, Hernandez-Guerra M, Romero R, Alonso I, González Y, Adrian Z, Hernandez G, Hernandez D, Delgado R, Quintero E. Impact of a 1-day versus 3-day low-residue diet on bowel cleansing quality before colonoscopy: a randomized controlled trial. Endoscopy. 2019;51(7):628-636. https://doi.org/10.1055/a-0864-1942
6. Repici A, Coron E, Sharma P, Spada C, Di Leo M, Noble CL, Gschossmann J, Bargalló García A, Baumgart DC. Improved high-quality colon cleansing with 1L NER1006 versus $2 \mathrm{~L}$ polyethylene glycol + ascorbate or oral sulfate solution. Dig Liver Dis. 2019;51(12):1671-1677. https://doi.org/10.1016/j.dld.2019.06.026

7. ASGE Standards of Practice Committee, Saltzman JR, Cash BD, Pasha SF, Early DS, Muthusamy VR, Khashab MA, Chathadi KV, Fanelli RD, Chandrasekhara V, Lightdale JR, Fonkalsrud L, Shergill AK, Hwang JH, Decker GA, Jue TL, Sharaf R, Fisher DA, Evans JA, Foley K, Shaukat A, Eloubeidi MA, Faulx AL, Wang A, Acosta RD. Bowel preparation before colonoscopy. Gastrointest Endosc. 2015;81(4):781-94. https://doi.org/10.1016/j.gie.2014.09.048

8. Murphy D, Jenks M, McCool R, Wood H, Young V, Amlani $\mathrm{B}$. A systematic review and cost analysis of repeat colonoscopies due to inadequate bowel cleansing in five European countries. Expert Rev Pharmacoecon Outcomes Res. 2019;19(6):701-709. https://doi.org/10.1080/14737167.2019.1597709

9. Rostom A, Dube C, Bishay K, Antonova L, Heitman SJ, Hilsden R. A randomized clinical prospective trial comparing split-dose picosulfate/ magnesium citrate and polyethylene glycol for colonoscopy preparation. PLoS 
One. 2019;14(3):e0211136.

https://doi.org/10.1371/journal.pone.0211136

10. Puckett J, Soop M. Optimizing colonoscopy preparation: the role of dosage, timing and diet. Curr Opin Clin Nutr Metab Care. 2012;15(5):499-504. https://doi.org/10.1097/MCO.0b013e328356b77b

11. Munsterman ID, Cleeren E, van der Ploeg T, Brohet R, van der Hulst R. 'Pico-Bello-Klean study': effectiveness and patient tolerability of bowel preparation agents sodium picosulphate-magnesium citrate and polyethylene glycol before colonoscopy. A single-blinded randomized trial. Eur J Gastroenterol Hepatol. 2015;27(1):29-38. https://doi.org/10.1097/MEG.0000000000000192

12. Klare P, Poloschek A, Walter B, Rondak IC, Attal S, Weber A, von Delius S, Bajbouj M, Schmid RM, Huber W. Singleday sodium picosulfate and magnesium citrate versus splitdose polyethylene glycol for bowel cleansing prior to colonoscopy: A prospective randomized endoscopist-blinded trial. J Gastroenterol Hepatol. 2015;30(11):1627-34. https://doi.org/10.1111/jgh.13010

13. Hassan C, East J, Radaelli F, Spada C, Benamouzig R, Bisschops R, Bretthauer M, Dekker E, Dinis-Ribeiro M, Ferlitsch M, Fuccio L, Awadie H, Gralnek I, Jover R, Kaminski MF, Pellisé M, Triantafyllou K, Vanella G, Mangas-Sanjuan C, Frazzoni L, Van Hooft JE, Dumonceau JM. Bowel preparation for colonoscopy: European Society of Gastrointestinal Endoscopy (ESGE) Guideline - Update 2019. Endoscopy. 2019;51(8):775-794.

https://doi.org/10.1055/a-0959-0505

14. Parente FR, Repici A, Crosta C, Cipolletta L, Testoni PA, Costamagna G, Andriulli A, Di Matteo G, Sassatelli R, Gallus S. Overall acceptability and efficacy of commonly used bowel preparations for colonoscopy in Italian clinical practice. A multicentre prospective study. Dig Liver Dis. 2014;46(9):795-802. https://doi.org/10.1016/j.dld.2014.05.002

15. Corporaal S, Kleibeuker JH, Koornstra JJ. Low-volume PEG plus ascorbic acid versus high-volume PEG as bowel preparation for colonoscopy. Scand J Gastroenterol. 2010;45(11):1380-6. https: / doi.org/10.3109/00365521003734158

16. Almadi MA, Alharbi O, Azzam N, Altayeb M, Thaniah $S$, Aljebreen A. Bowel preparation quality between hospitalized patients and outpatient colonoscopies. Saudi J Gastroenterol. 2018;24(2):93-99. https://doi.org/10.4103/sjg.SJG_485_17

17. Argyropoulos SK, Mahmood SK, Campbell EJ, Richter JM. Improving the Quality of Inpatient Bowel Preparation for Colonoscopies. Dig Dis Sci. 2018;63(2):338-344. https://doi.org/10.1007/s10620-017-4896-0

18. Asociación Médica Mundial. Declaración de Helsinki de la AMM - Principios éticos para las investigaciones médicas en seres humanos [Internet]. Universidad de Navarra; 2013 [consultado el 18 de septiembre de 2020]. Disponible en: http://www.redsamid.net/archivos/201606/2013-declaracion-helsinki-brasil.pdf?1
19. Resolución número 8430 de 1993, Por la cual se establecen las normas científicas, técnicas y administrativas para la investigación en salud [Internet]. Ministerio de Salud de Colombia; 1993 [consultado el 22 de septiembre de 2020]. Disponible en: https://www.minsalud.gov.co/sites/rid/ Lists/BibliotecaDigital/RIDE/DE/DIJ/RESOLUCION8430-DE-1993.PDF

20. Sweetser S, Baron TH. Optimizing bowel cleansing for colonoscopy. Mayo Clin Proc. 2015;90(4):520-6. https://doi.org/10.1016/j.mayocp.2015.01.015

21. Hassan C, Fuccio L, Bruno M, Pagano N, Spada C, Carrara S, Giordanino C, Rondonotti E, Curcio G, Dulbecco P, Fabbri C, Della Casa D, Maiero S, Simone A, Iacopini F, Feliciangeli G, Manes G, Rinaldi A, Zullo A, Rogai F, Repici A. A predictive model identifies patients most likely to have inadequate bowel preparation for colonoscopy. Clin Gastroenterol Hepatol. 2012;10(5):501-6. https://doi.org/10.1016/j.cgh.2011.12.037

22. Doykov D, Andonov V. Risk Factors and Incidence of Poor Bowel Preparation in Elderly Patients: Prospective Study. Folia Med (Plovdiv). 2019;61(3):370-376. https://doi.org/10.3897/folmed.61.e39409

23. Padmanabhan H, Rothnie A, Higgins A, Grewal A, Arndtz K, Nevill AM, Brookes MJ, Mathew R. Preassessment Interview Improves the Efficacy and Safety of Bowel Preparation for Colonoscopy. Can J Gastroenterol Hepatol. 2016;2016:7591637. https://doi.org/10.1155/2016/7591637

24. Guo X, Li X, Wang Z, Zhai J, Liu Q, Ding K, Pan Y. Reinforced education improves the quality of bowel preparation for colonoscopy: An updated meta-analysis of randomized controlled trials. PLoS One. 2020;15(4):e0231888. https://doi.org/10.1371/journal.pone.0231888

25. Sim JS, Koo JS. Predictors of Inadequate Bowel Preparation and Salvage Options on Colonoscopy. Clin Endosc. 2016;49(4):346-9. https://doi.org/10.5946/ce.2016.094

26. Shah HA, Paszat LF, Saskin R, Stukel TA, Rabeneck L. Factors associated with incomplete colonoscopy: a population-based study. Gastroenterology. 2007;132(7):2297-303. https://doi.org/10.1053/j.gastro.2007.03.032

27. Martens P, Bisschops R. Bowel preparation for colonoscopy: efficacy, tolerability and safety. Acta Gastroenterol Belg. 2014;77(2):249-55.

28. Jin Z, Lu Y, Zhou Y, Gong B. Systematic review and meta-analysis: sodium picosulfate/magnesium citrate vs. polyethylene glycol for colonoscopy preparation. Eur J Clin Pharmacol. 2016;72(5):523-32. https://doi.org/10.1007/s00228-016-2013-5

29. Gweon TG, Kim SW, Noh YS, Hwang S, Kim NY, Lee Y, Lee SW, Lee SW, Lee JY, Lim CH, Hun Kim H, Kim JS, Kyung Cho Y, Myung Park J, Seok Lee I, Myung-Gyu Choi. Prospective, randomized comparison of sameday dose of 2 different bowel cleanser for afternoon colonoscopy: picosulfate, magnesium oxide, and citric acid versus polyethylene glycol. Medicine (Baltimore). 
2015;94(13):e628.

https://doi.org/10.1097/MD.0000000000000628

30. Jeon SR, Kim HG, Lee JS, Kim JO, Lee TH, Cho JH, Kim YH, Cho JY, Lee JS. Randomized controlled trial of lowvolume bowel preparation agents for colonic bowel preparation: 2-L polyethylene glycol with ascorbic acid versus sodium picosulfate with magnesium citrate. Int J Colorectal Dis. 2015;30(2):251-8. https://doi.org/10.1007/s00384-014-2066-9

31. Kang MS, Kim TO, Seo EH, Jung DK, Kim MS, Heo NY, Park JH, Park SH, Moon YS. Comparison of the Efficacy and Tolerability between Same-day Picosulfate and Splitdose Polyethylene Glycol Bowel Preparation for Afternoon Colonoscopy: A Prospective, Randomized, Investigatorblinded Trial. Intest Res. 2014;12(1):53-9. https://doi.org/10.5217/ir.2014.12.1.53

32. Lawrance IC, Willert RP, Murray K. Bowel cleansing for colonoscopy: prospective randomized assessment of efficacy and of induced mucosal abnormality with three preparation agents. Endoscopy. 2011;43(5):412-8. https://doi.org/10.1055/s-0030-1256193

33. Kim YS, Hong CW, Kim BC, Han KS, Park JW, Seong Choi $\mathrm{H}$, Joo J, Sohn DK. Randomized clinical trial comparing reduced-volume oral picosulfate and a prepackaged lowresidue diet with 4-liter PEG solution for bowel preparation. Dis Colon Rectum. 2014;57(4):522-8. https://doi.org/10.1097/DCR.0000000000000066

34. Manes G, Amato A, Arena M, Pallotta S, Radaelli F, Masci E. Efficacy and acceptability of sodium picosulphate/ magnesium citrate vs low-volume polyethylene glycol plus ascorbic acid for colon cleansing: a randomized controlled trial. Colorectal Dis. 2013;15(9):1145-53. https://doi.org/10.1111/codi.12246

35. Nam SJ, Kim YJ, Keum B, Lee JM, Kim SH, Choi HS, Kim ES, Seo YS, Jeen YT, Lee HS, Chun HJ, Um SH, Kim CD. Impact of diet restriction on bowel preparation for colonoscopy. Medicine (Baltimore). 2018;97(41):e12645. https://doi.org/10.1097/MD.0000000000012645

36. Song GM, Tian X, Ma L, Yi LJ, Shuai T, Zeng Z, Zeng XT. Regime for Bowel Preparation in Patients Scheduled to Colonoscopy: Low-Residue Diet or Clear Liquid Diet? Evidence From Systematic Review With Power Analysis. Medicine (Baltimore). 2016;95(1):e2432. https://doi.org/10.1097/MD.0000000000002432

37. Labuschagne GS, Morris RW. The effect of oral intake during the immediate pre-colonoscopy time period on volume depletion in patients who receive sodium picosulfate. Anaesth Intensive Care. 2017;45(4):485-489. https://doi.org/10.1177/0310057X1704500412

38. Holte K, Nielsen KG, Madsen JL, Kehlet H. Physiologic effects of bowel preparation. Dis Colon Rectum. 2004;47(8):1397-402. https://doi.org/10.1007/s10350-004-0592-1

39. Kojecky V, Matous J, Keil R, Dastych M, Kroupa R, Zadorova Z, Varga M, Dolina J, Kment M, Hep A. A head-to-head comparison of 4-L polyethylene glycol and low-volume solutions before colonoscopy: which is the best? A multicentre, randomized trial. Int J Colorectal Dis. 2017;32(12):1763-1766. https://doi.org/10.1007/s00384-017-2901-x

40. Martel M, Barkun AN, Menard C, Restellini S, Kherad O, Vanasse A. Split-Dose Preparations Are Superior to Day-Before Bowel Cleansing Regimens: A Meta-analysis. Gastroenterology. 2015;149(1):79-88. https://doi.org/10.1053/j.gastro.2015.04.004

41. Kwak MS, Cha JM, Yang HJ, Park DI, Kim KO, Lee J, Shin JE, Joo YE, Park J, Byeon JS, Kim HG. Safety and Efficacy of Low-Volume Preparation in the Elderly: Oral Sulfate Solution on the Day before and Split-Dose Regimens (SEE SAFE) Study. Gut Liver. 2019;13(2):176-182. https://doi.org/10.5009/gnl18214

42. Mohamed R, Hilsden RJ, Dube C, Rostom A. SplitDose Polyethylene Glycol Is Superior to Single Dose for Colonoscopy Preparation: Results of a Randomized Controlled Trial. Can J Gastroenterol Hepatol. 2016;2016:3181459. https://doi.org/10.1155/2016/3181459

43. Church JM. Effectiveness of polyethylene glycol antegrade gut lavage bowel preparation for colonoscopy--timing is the key! Dis Colon Rectum. 1998;41(10):1223-5. https://doi.org/10.1007/BF02258217

44. Tandon K, Khalil C, Castro F, Schneider A, Mohameden M, Hakim S, Shah K, To C, O’Rourke C, Jacobs J. Safety of Large-Volume, Same-Day Oral Bowel Preparations During Deep Sedation: A Prospective Observational Study. Anesth Analg. 2017;125(2):469-476.

https://doi.org/10.1213/ANE.0000000000001805

45. Cohen LB. Split dosing of bowel preparations for colonoscopy: an analysis of its efficacy, safety, and tolerability. Gastrointest Endosc. 2010;72(2):406-12. https://doi.org/10.1016/j.gie.2010.04.001

46. McNabb-Baltar J, Dorreen A, Al Dhahab H, Fein M, Xiong X, O’ Byrne M, Ait I, Martel M, Barkun AN. Age Is the Only Predictor of Poor Bowel Preparation in the Hospitalized Patient. Can J Gastroenterol Hepatol. 2016;2016:2139264. https://doi.org/10.1155/2016/2139264

47. Barkun A, Chiba N, Enns R, Marcon M, Natsheh S, Pham C, Sadowski D, Vanner S. Commonly used preparations for colonoscopy: efficacy, tolerability, and safety--a Canadian Association of Gastroenterology position paper. Can J Gastroenterol. 2006;20(11):699-710. https://doi.org/10.1155/2006/915368

48. Lichtenstein GR, Cohen LB, Uribarri J. Review article: Bowel preparation for colonoscopy--the importance of adequate hydration. Aliment Pharmacol Ther. 2007;26(5):633-41. https://doi.org/10.1111/j.1365-2036.2007.03406.x

49. Lorenzo-Zúñiga V, Moreno-de-Vega V, Boix J. Preparación para colonoscopia: tipos de productos y escalas de limpieza. Rev Esp Enferm Dig. 2012;104(8):426-31. https://doi.org/10.4321/S1130-01082012000800006 